\title{
Fast Fourier transform-based homogenisation of gas hydrate bearing sediments
}

\author{
A. ALAVOINE* , P. DANGLA* and J.-M. PEREIRA*
}

\begin{abstract}
Modelling the mechanical response of gas hydrate bearing sediments has become a crucial issue in the environmental field. The scarcity of experimental data due to the instability of the gas hydrates makes it difficult to develop accurate mechanical constitutive models. This paper suggests a numerical homogenisation method to simulate the response of composite materials like gas hydrate bearing soils. The homogenisation technique is based on Fast Fourier Transforms and thus can be used with real images of soils. This method is applied here to both granular and fine soils for various volume fractions and types of gas hydrate inclusions.
\end{abstract}

KEYWORDS: constitutive relations; clays; sands; shear strength; elasticity; plasticity; numerical modelling

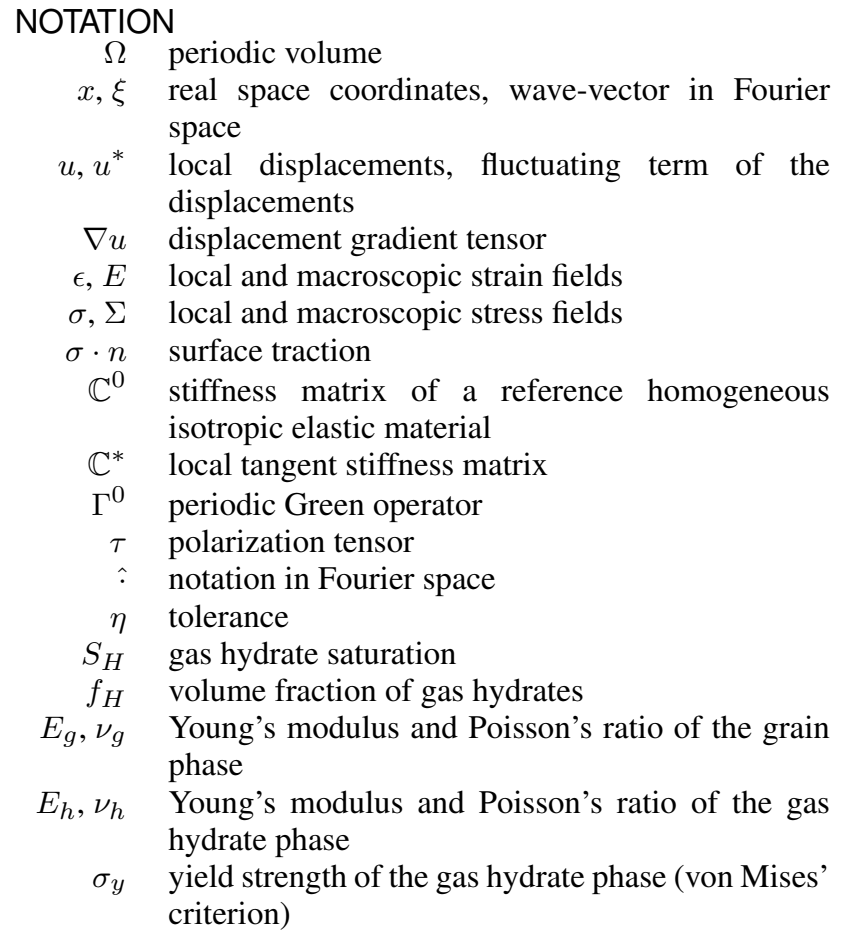

\section{INTRODUCTION}

Gas hydrates are crystalline compounds combining hydrogenbonded water molecules and gas molecules. Natural gas hydrates form under high pressure and low temperature conditions in oceanic sediments or permafrost regions. They originate essentially from methane gas that is mainly a decomposition product of organic matter. These gas hydrate bearing sediments (GHBS) are a potential energy resource but their instability also raises environmental concerns, which is why it became important to understand and predict their mechanical behaviour. Even though ocean exploration

\footnotetext{
Manuscript received...

Published online at www.geotechniqueletters.com

* Navier, École des Ponts, Univ Gustave Eiffel, CNRS, Marne-laVallée, France
}

programs have led to numerous scientific insights, core samples remain difficult to obtain without dissociating the hydrates. Experiments have been performed mostly on samples synthesised in the laboratory (Masui et al., 2005; Miyazaki et al., 2011; Hyodo et al., 2013; Kato et al., 2016), but also on some pressure core samples (Santamarina et al., 2015; Yoneda et al., 2015). The results showed the effect of the gas hydrates inclusions on the mechanical response of the sediments. Depending on the volume fraction of the hydrates phase, the type of host sediment, and on the pore habit, the strength and stiffness parameters of the sediments vary (Waite et al., 2009; Soga et al., 2006). For example, the shear strength and cohesion of GHBS tend to increase with an increasing volume fraction of gas hydrates, beyond a threshold value of this ratio (Waite et al., 2009). However it is still difficult to systematically characterise the mechanical behaviour of these soils under in situ conditions considering the heterogeneity of their structure, the number of different influential parameters, and the cost of experiments and sample recovery.

Homogenisation techniques can be a way to overcome these experimental difficulties and to study the impact of the gas hydrate phase on the apparent mechanical response of a heterogeneous microstructure. Numerical homogenisation include a good range of methods that can apply -assuming periodic cells- to complex microstructures, composed of constituents characterised by linear or non-linear mechanical behaviour (including elastoplastic behaviour). In particular, an original method based on the use of Fast Fourier Transforms (FFT) in a fixed point iteration algorithm (Moulinec \& Suquet, 1994, 1998) to solve the local mechanical problem of a periodic composite cell has inspired different computational schemes (Michel et al., 2000; Brisard \& Dormieux, 2010; Monchiet \& Bonnet, 2013). An advantage of FFT-based methods is that they do not need any mesh, and could instead use real images of microstructures. However one of the main limitations of the original method, that concerns GHBS, is the slow or even non-convergence in case of high stiffness contrast between phases, like when voids or rigid inclusions are present in the microstructure (Moulinec \& Suquet, 1998). Among the new FFT-based homogenisation schemes many authors chose Krylov subspace solvers to improve convergence and solve the stiffness contrast issue (Brisard \& Dormieux, 2010; Zeman et al., 2010). 
This paper aims at studying the application of a FFTbased numerical homogenisation scheme to model the mechanical behaviour of different microstructures of GHBS. The computational framework used in the present work and initially developed by Gélébart \& Mondon-Cancel (2013) is first recalled. Then, the framework is applied to granular and fine host sediments examples for different gas hydrates pore habits and volume fractions. Finally, a real image acquired from micro CT scans of GHBS from Krishna-Godavari Basin (Rees et al., 2011) is homogenised with the same algorithm to show its capabilities.

\section{NUMERICAL METHOD}

In this section we present the different methods that we have combined in an in-house numerical homogenisation code. We based our code on the numerical methods developed by Moulinec \& Suquet (1994) and Gélébart \& Mondon-Cancel (2013), and we implemented the local integration of some nonlinear models like the modified Cam Clay criterion.

\section{FFT-based homogenisation}

In periodic homogenisation the global response of a composite material subjected to a macroscopic strain $E$ is obtained via the resolution of the local mechanical problem on a representative volume element $\Omega$, with periodic boundary conditions. The unit cell is defined by a non-uniform microscopic constitutive law $F$ relating the local stress field $\sigma$ to the local strain field $\epsilon$. The governing equations are as follows:

$$
\begin{cases}\operatorname{div}(\sigma(x))=0 & (x \in \Omega) \\ \sigma(x)=F(\epsilon(x)) & (x \in \Omega) \\ 2 \epsilon(x)=\left(\nabla u(x)+\nabla^{T} u(x)\right) & (x \in \Omega) \\ u(x)=E \cdot x+u^{*}(x) & (x \in \Omega) \\ u^{*}(x) \text { periodic } & \text { (opposite boundaries) } \\ \sigma \cdot n \text { antiperiodic } & \text { (opposite boundaries) }\end{cases}
$$

where the local displacements $u$ are split into their average value $E \cdot x$ and a periodic fluctuating term $u^{*}(x)$, and are linked to the local strains $\epsilon$ by the compatibility equation. The macroscopic stress $\Sigma$ can be obtained by averaging the local stress field $\sigma$ over the unit cell. Moulinec \& Suquet (1994) introduced a reference elastic homogeneous material of stiffness tensor $\mathbb{C}^{0}$ and a polarisation tensor $\tau(x)=$ $\left(\mathbb{C}(x)-\mathbb{C}^{0}\right): \epsilon(x)$ to reformulate the problem and express it as the search for the solution of the periodic LippmannSchwinger equation in real and Fourier spaces, respectively:

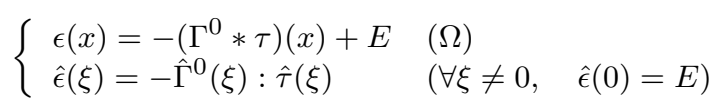

with $\Gamma^{0}$ being the periodic Green operator associated to $\mathbb{C}^{0}$, $\xi$ the wave-vector in Fourier space, and $*$ the convolution product. The latter advantageously becomes a direct product in Fourier space, and offers the possibility to solve the integral equation through different techniques involving Fourier transforms. In the 'basic scheme', Moulinec \& Suquet (1998) discretised the unit cell into a grid of pixels (2D) or voxels (3D), and the problem equations in order to use the Fast Fourier Transforms (FFT). They then proposed a fixed point algorithm to find the solution of the Lippmann-Schwinger equation, and were followed by many authors that developed other solving techniques.

\section{Newton-Raphson algorithm}

The method adopted here differs from Moulinec and Suquet original work by its resolution method. We use the same one as
Gélébart \& Mondon-Cancel (2013). It makes use of a general Newton-Raphson (NR) algorithm to solve the LippmannSchwinger equation with the following residual:

$$
\mathcal{G}(\epsilon(t))=\epsilon(t)+\Gamma^{0} *\left(\sigma(t)-\mathbb{C}^{0}: \epsilon(t)\right)-E(t)
$$

We use the discrete Green operator from Moulinec \& Suquet (1994) here. Non-linear constitutive laws can be distributed over the unit cell. The stresses and internal variables need to be integrated over time increments $\Delta t=t_{n}-t_{n-1}$, as a function of the previous state. The Jacobian matrix $\frac{\partial \mathcal{G}}{\partial \epsilon}\left(\epsilon\left(t_{n}\right)\right)$ is not given since the residual differentiation includes a convolution product, but the linearised problem in the NR algorithm is fully expressed as follows:

$$
\begin{gathered}
\frac{\partial \mathcal{G}}{\partial \epsilon}\left(\epsilon\left(t_{n}\right)\right): \delta \epsilon=-\mathcal{G}\left(\epsilon\left(t_{n}\right)\right) \\
\Longleftrightarrow \delta \epsilon+\Gamma^{0} *\left(\left(\mathbb{C}^{*}-\mathbb{C}^{0}\right): \delta \epsilon\right)=-\mathcal{G}\left(\epsilon\left(t_{n}\right)\right)
\end{gathered}
$$

and solved by a Conjugate Gradient (CG) method to find $\delta \epsilon$ and update the strains at iteration $k: \epsilon\left(t_{n}\right)^{k}=\epsilon\left(t_{n}\right)^{k-1}+\delta \epsilon . \mathbb{C}^{*}$ is the 'tangent stiffness matrix':

$$
\mathbb{C}^{*}=\frac{\partial \sigma\left(t_{n}\right)}{\partial \epsilon}\left(\epsilon\left(t_{n}\right)\right)
$$

and depends on the constitutive law. The convergence criterion used in the NR algorithm is the stress equilibrium condition evaluated in Fourier space at each iteration (Moulinec \& Suquet, 1998; Gélébart \& Mondon-Cancel, 2013):

$$
\frac{\left(\left\langle\left\|\operatorname{div}\left(\sigma\left(t_{n}\right)\right)\right\|^{2}\right\rangle\right)^{1 / 2}}{\left\|\left\langle\sigma\left(t_{n}\right)\right\rangle\right\|}<\eta
$$

In our calculations the CG-based solver comes from the PETSc (Balay et al., 2019) library, and the convergence criterion is applied outside the framework of the library, in addition to its internal one. This criterion is the same equilibrium condition as equation 7 but evaluated for the tangential stresses that are extrapolated from the CG loop solution (Gélébart \& MondonCancel, 2013):

$$
\sigma^{C G}=\sigma\left(t_{n-1}\right)+\mathbb{C}^{*}: \delta \epsilon
$$

We compared our FFT-based homogenisation with a FEM simulation code developed in our laboratory in order to validate our simulation method. A good agreement is observed (figure 1).

In order to model the effective mechanical behaviour of GHBS, one has to take into account not only the volume fraction of the gas hydrate phase in the sedimentary material, but also the complex geometries of these compounds. Furthermore, the observed non-linear response of sheared GHBS specimens seems to be strongly related to the nonlinear behaviour of the microscopic components, in particular of the gas hydrate phase. The use of homogenisation techniques accounting for simple elastoplasticity allows us to integrate these non-linearities based on realistic geometries of the microstructure. Working directly at the macroscopic level using non-linear constitutive laws to capture the observed behaviour of GHBS would certainly require a significant increase of the number of material parameters.

\section{APPLICATION TO GAS HYDRATE BEARING SEDIMENTS}

Gas hydrate crystals occupy the pore space of sediments in different ways depending on the conditions under which they 
(a) FEM, $\mathrm{E}_{12}=0.8 \%$

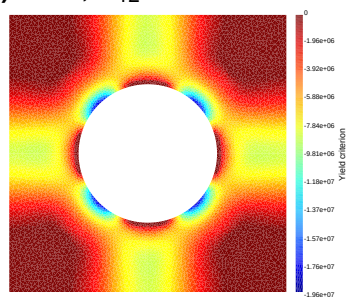

(c) FFT, $\mathrm{E}_{12}=0.8 \%$

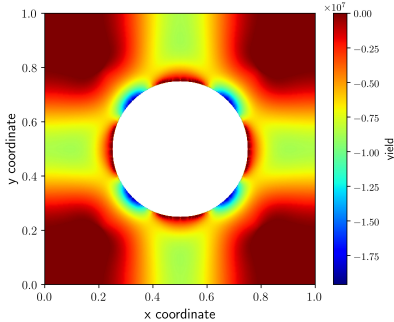

(b) FEM, $\mathrm{E}_{12}=1 \%$

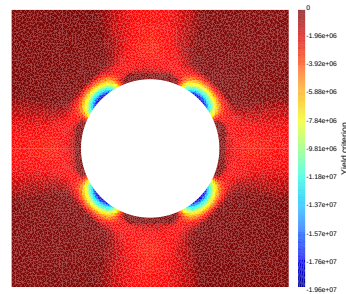

(d) FFT, $E_{12}=1 \%$

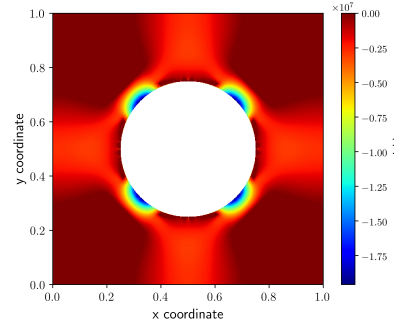

Fig. 1. Example of comparative results of periodic homogenisation simulations of a shear loading $\left(E_{12}=1 \%, E_{11}=E_{22}=0\right)$ applied to a circular elastic inclusion in an elastoplastic matrix using FEM (top figures, mesh of 7216 elements) or FFT (bottom figures, $512 \times 512$ pixels). All figures represent the distribution of the yield function over the matrix phase at two different values of the macroscopic strain load: $E_{12}=0.8 \%$ (left) and $E_{12}=1 \%$ (right).

were formed (Waite et al., 2009). In coarse-grained sediments gas hydrates can occur under various types of pore habits usually described in the literature as pore-filling, load-bearing or cementing (Waite et al., 2009; Chaouachi, 2016). It is generally assumed that in the laboratory one of the two common methods of synthesis leads to a load-bearing type of hydrates while the other one leads to a cementing type of hydrates (Ebinuma et al., 2005). In fine sediments they can fracture the soil matrix and form networks of veins (Rees et al., 2011). These complex microstructures influence the macroscopic (apparent) response of the material. The FFTbased homogenisation method we described in the previous section can be applied to study the impact of parameters such as pore habits or volume fraction of gas hydrates on the macroscopic behaviour of GHBS. We specify that this method works well both in 2D and 3D.

\section{Effect of pore habits in granular soils}

In this section, different periodic geometries of twodimensional coarse-grained microstructures were tested as examples (see figure 2 and figure 3 ) to demonstrate the relevance of the method in the study of gas hydrate bearing soils. The pore space around randomly distributed grains at a given porosity was filled with hydrates using two different scenarios of pore habits: load-bearing and cementing gas hydrates (see figure 3 ). Three phases constitute the material in these calculations: rigid grains, hydrates and liquid, with a volume fraction of gas hydrates varying between $80 \%$ and $100 \%$ of the pore space. We did not consider lower hydrate saturations in these simulations in $2 \mathrm{D}$ because we needed a surface of hydrates large enough to ensure a continuity between the solid phases to avoid the occurrence of floating grains. A loading corresponding to an oedometer test was applied to the different unit cells:

$$
\Delta E_{11}=1 \% \text { and } \Delta E_{22}=\Delta E_{12}=\Delta E_{33}=0
$$
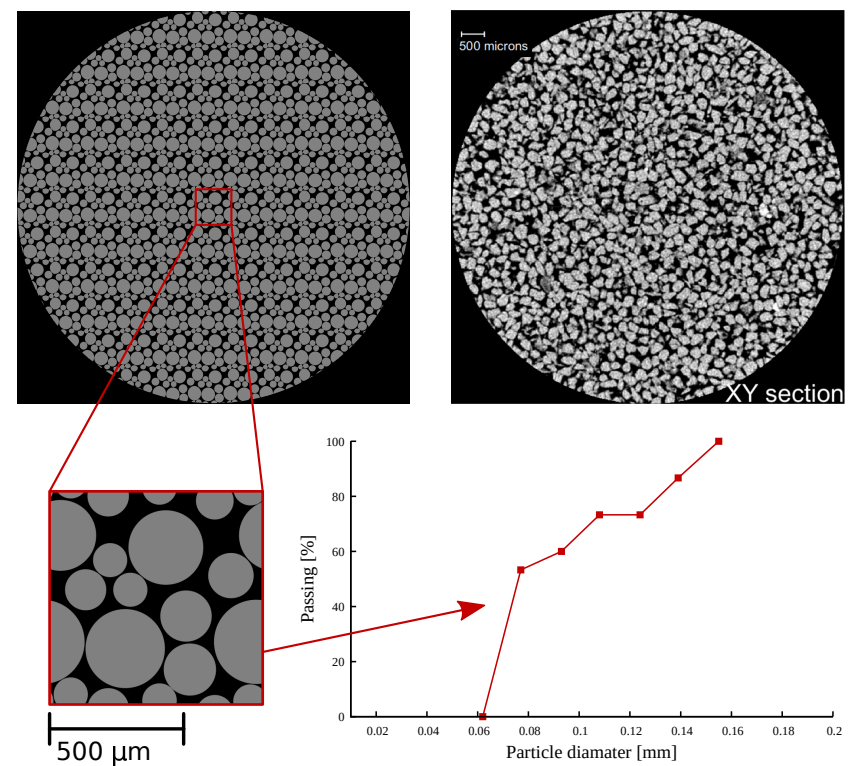

Fig. 2. The upper right image shows a typical example of a real coarse-grained microstructure with a porosity of $n=0.28$ (Al Mahbub \& Haque, 2016) and the upper left image represents an example of the ideal periodic microstructures that we imagined for our calculations. The unit cell used in this example to describe the microstructure (with a porosity of $n=0.27$ ) is shown below. Here we only represent the granular skeleton of the microstructure, obtained by randomly adding circles with a radius between 0.08 and 0.2 in a unit square. To obtain realistic values of microstructures from this unit-square, we simply multiply the length of each geometrical object by the desired length scale, which gives us the realistic grain size distribution on the right.

The grains were assumed to have an isotropic linear elastic behaviour, defined by the following Young's modulus and Poisson's ratio: $E_{g}=2.45 \mathrm{GPa}, \nu_{g}=0.1$. We used the Poisson's ratio of the quartz material (Mcknight et al., 2008), and we chose a Young's modulus that gave us results approaching those of mechanical tests performed on methane hydrate bearing sands in the laboratory (Le et al., 2019). There are only few data and test results regarding the behaviour of pure gas hydrates samples, but Yoneda et al. (2019) managed to retrieve natural pressure core samples of massive gas hydrates and tested them. They obtained values for the Young's modulus of $287 \mathrm{MPa}$ and $350 \mathrm{MPa}$ under quasi-static conditions, and maximum deviator stress values of $3.04 \mathrm{MPa}$ and 3.24 MPa. This is why the gas hydrate phase was defined by an elastic perfectly plastic model with von Mises' criterion, with the given parameters: $E_{h}=300 \mathrm{MPa}, \nu_{h}=0.32$, and a yield strength equal to $\sigma_{y}=3 \mathrm{MPa}$. The Poisson's ratio for the pure hydrate phase was taken from Sloan \& Koh (2007). The voids represent fluid-filled porosity and were characterised by a null stiffness matrix, like in a drained test configuration, as we homogenise the effective properties of the material. The macroscopic stress-strain response is obtained by averaging the microscopic fields over the entire unit cell at the end of each time step. A tolerance of $\eta=10^{-7}$ was taken for the calculations.

First, we looked at the repeatability of our simulations by performing calculations over randomly drawn unit cells with a hydrate saturation of $100 \%$ and a porosity between $27.8 \%$ and $28.8 \%$ but with different arrangements of the granular skeleton. The mean macroscopic response and the corresponding standard deviation obtained for 10 different 


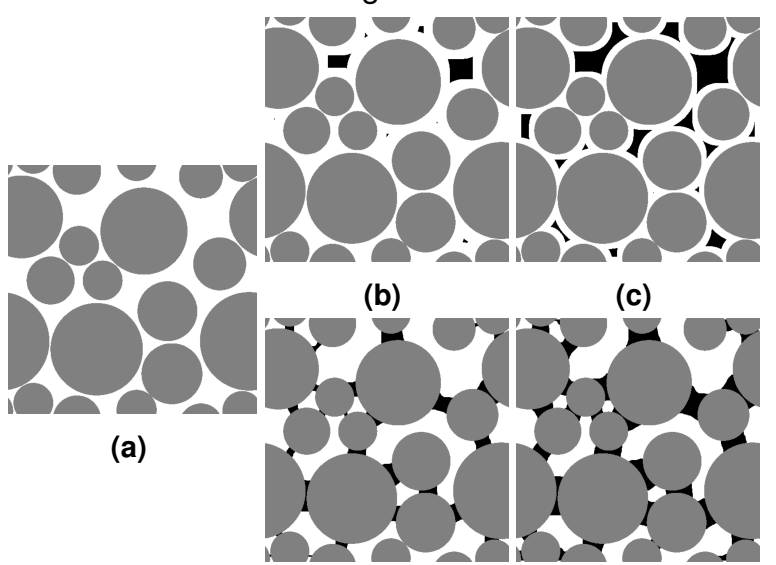

(d)

(e)

Fig. 3. Examples of microstructures for a porosity of $n=0.27$ and at a resolution of 512 pixels, with solid grains in grey, gas hydrates in white and voids in black. In 3 a voids are completely filled with hydrates. $3 b$ and $3 c$ represent cementing gas hydrates at $95 \%$ and $80 \%$ of pore volume fraction respectively while $3 d$ and 3 e represent load-bearing gas hydrates at $90 \%$ and $80 \%$ of pore volume fraction.

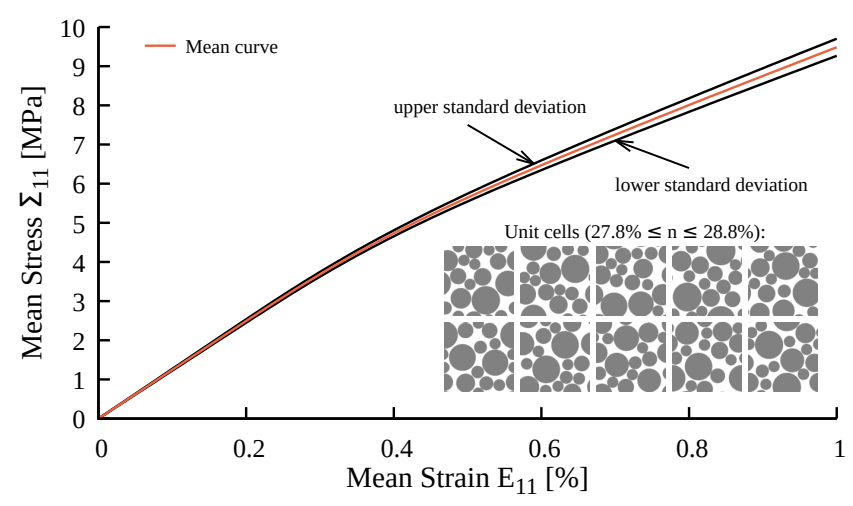

Fig. 4. Average macroscopic stress-strain response (in red) to the oedometer loading of 10 random granular microstructures, each with a porosity $n$ between $27.8 \%$ and $28.8 \%$. The upper and lower black curves represent the standard deviation of the responses of the simulated microstructures that are represented at the bottom right part of the figure.

simulations are plotted in figure 4 and show a rather low dispersion.

Figure 5 shows the results for 'cemented' microstructures of two different porosities, $n=0.4$ and $n=0.27$, and various values of volume fraction of gas hydrates for each of them. As expected, the increase in gas hydrate saturation and the decrease in porosity lead to a stiffer material, which is in agreement with many experimental results. In the figure 6, a comparison between the effects of cementing and loadbearing gas hydrates is represented for a given porosity. The global responses of the two types of pore habits for the same volume fraction of hydrates match expectations. The cemented structures have a stiffer mechanical response while the loadbearing structure yields more rapidly. Both morphologies reach a perfectly plastic behaviour at $\mathrm{S}_{\mathrm{H}}=80 \%$. The fact that the chosen technique does not take into account contact laws and that we chose a unit cell containing only few grains plays a role on the resulting macromechanical fields. The geometries that were chosen as unit cells in this section are based on circular solid grains representing the sedimentary phase, but more complex and realistic microstructures have to be used in the study of gas hydrate bearing sediments. The advantage of

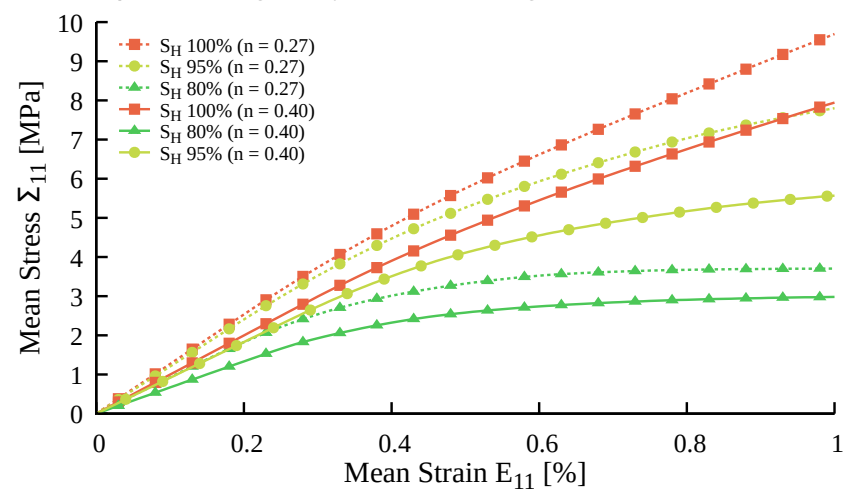

Fig. 5. Macroscopic stress-strain response to the loading of the granular soil with cementing gas hydrates for different values of porosity and gas hydrate saturation, at a resolution of 512 pixels.

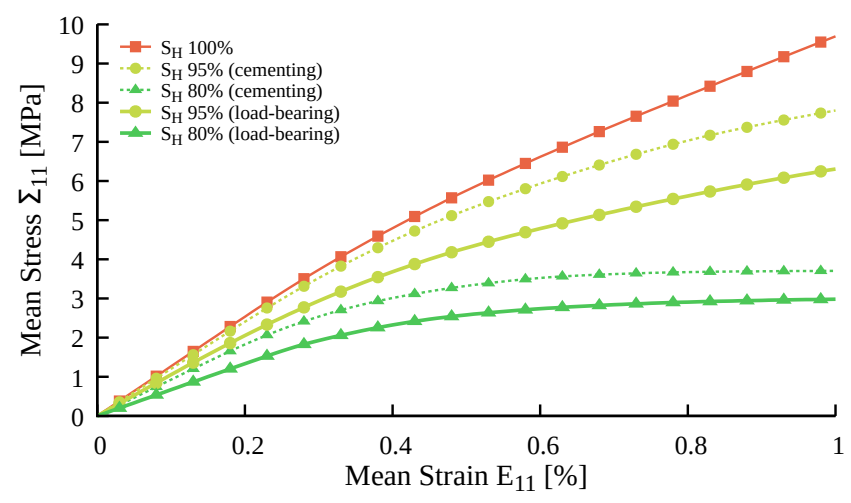

Fig. 6. Macroscopic stress-strain response to an 'oedometer' loading of granular soil for different pore habits - cementing and load-bearing - and different values of gas hydrate saturation $\mathrm{S}_{\mathrm{H}}$, at a porosity of $n=0.27$ and a resolution of 512 pixels.

the FFT-based homogenisation methods is that one can analyse real images. In the next section we present the case of fine soils containing gas hydrate with unit cells adapted from real images.

\section{Using real images}

Few studies managed for now to get real images of gas hydrate bearing soils at the microscopic scale. Since it is difficult and expensive to get pressure core samples and maintain them under in situ conditions, the observation of gas hydrates nucleation through x-ray microtomography for example are mainly done with synthetic samples (Chaouachi, 2016). However the investigations that have been conducted until today give an insight of the actual microstructure of gas hydrate bearing soils and can be used as unit cells to homogenise the mechanical properties.

In this section we applied the same FFT-based homogenisation to a real image of hydrate veins in fine sediments. There are only few images of the microstructure of fine-grained sediments with gas hydrates. It is known that gas hydrates tend to occur as veins and nodules in fine-grained sediments, in contrast with coarse-grained soils. A unit cell with a resolution of 256 pixels was defined based on the micro CT scans of depressurised and cooled cores from the Krishna-Godavari Basin (Rees et al., 2011) represented in figure 7 . The figure 8 shows the original image and its periodic-plus-smooth decomposition (Moisan, 2011) via Brisard's Python implementation (Brisard, 2018). By working with a non-periodic image the resulting mechanical fields would be disturbed at the edges. This image processing allows us to work with a periodic REV, but it should be noted that it generates a disturbed image from the original one 


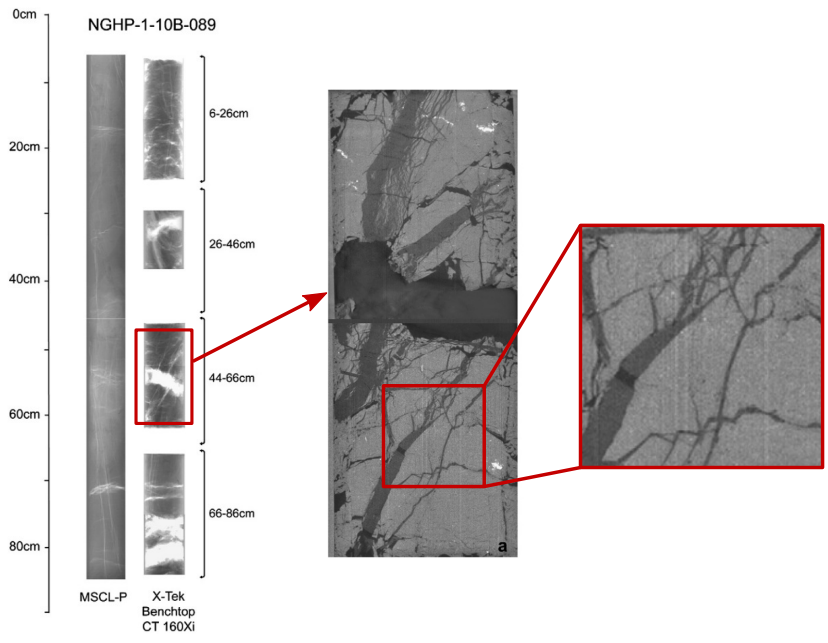

Fig. 7. On the left are represented X-ray images of a natural core published by Rees et al. (2011) and from which the vertical slice (a, in the middle) of core section $46-66 \mathrm{~cm}$ was taken using the micro CT scanning method. On the right we represent the extracted image that we chose to use for our application. The image corresponds to an area with vein inclusions, while we can observe a nodule above it in the middle image.

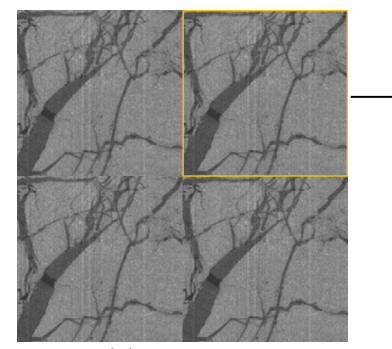

(a) Original

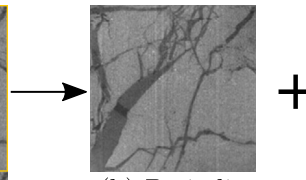

(b) Periodic

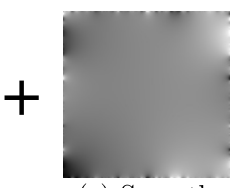

(c) Smooth

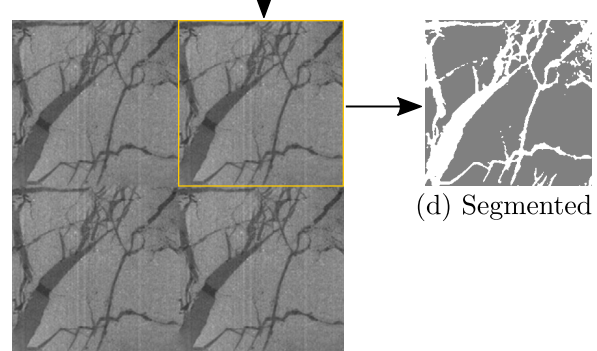

Fig. 8. Periodic pattern made with the real image (a) used as a unit cell (yellow box - 256 pixels resolution); its periodic (b) plus smooth (c) decomposition and the segmented image (d) used for calculations.

around the edges. Considering the sample disturbance prior to scanning, in this example we chose to define only two phases from the periodic microstructure: the fine-grained soil matrix and the gas hydrates phase forming veins (figure 8d). Two threshold values were tested for the image segmentation: (A) the minimum value of the valley between the two principal peaks of the image histogram, and (B) the average value of these two peaks (figure 9). Morphological operations such as dilation and erosion were then applied to process the resulting images in order to get artificial volume fractions of the gas hydrate phase $f_{\mathrm{H}}(14 \%, 16 \%, 23 \%$ and $34 \%)$. These operations consist in adding or removing pixels to or from object boundaries, the object being hydrates veins in our case.

The gas hydrate phase was defined by an elastic perfectly plastic model with von Mises' criterion, with the given elastic parameters: $E_{h}=9 \mathrm{GPa}, \nu_{h}=0.32$ (dynamic moduli from Sloan \& Koh, 2007). For a first assessment and based on
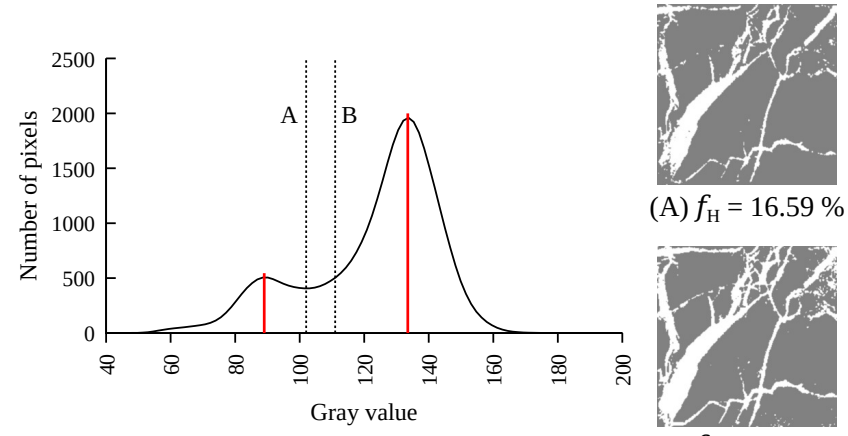

(A) $f_{\mathrm{H}}=16.59 \%$

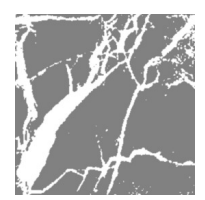

(B) $f_{\mathrm{H}}=23.63 \%$

Fig. 9. On the left the smooth histogram of the periodic real image (see figure $8 \mathrm{~b}$ ) from which two threshold values were chosen: $A$ is the grey value corresponding to the minimum number of pixels in the valley between the two peaks in red, and $B$ is the mean value of these peaks. On the right the segmented images obtained from $A$ and $B$ and the corresponding volume fraction of gas hydrates $f_{\mathrm{H}}$.

Table 1. Cam Clay properties for the fine soil matrix (Torisu et al., 2012)

\begin{tabular}{|lrl|}
\hline Parameter & Value & Unit \\
\hline Compression index $\lambda$ & 0.1 & - \\
Swelling index $\kappa$ & 0.01 & - \\
Elastic shear modulus $G$ & 300 & $(\mathrm{MPa})$ \\
Pre-consolidation pressure $p_{c 0}$ & 2 & $(\mathrm{MPa})$ \\
Initial Porosity $\phi$ & 0.37 & - \\
Slope of critical state line $M$ & 1.0 & - \\
Initial mean stress $p_{0}$ & 1.25 & $(\mathrm{MPa})$ \\
\hline
\end{tabular}

existing data (Hyodo et al., 2002; Yu et al., 2011) the yield strength has been taken equal to $\sigma_{y}=5 \mathrm{MPa}$. The material for the soil matrix was defined by a modified Cam-Clay model, and the properties are summarised in the table 1. The macroscopic loading consisted in a mixed loading, comparable to a triaxial compression test loading. Starting from a uniform initial stress of $1.25 \mathrm{MPa}$ over the entire cell, the horizontal stresses were kept constant while a vertical strain loading was imposed until reaching the macroscopic mean strain value of $5 \%$ :

$$
\Delta E_{11}=5 \% \text { and } \Delta \Sigma_{22}=\Delta \Sigma_{33}=\Delta \Sigma_{i j}=0 \quad \forall i \neq j
$$

Given the fact that the unit cell is two-dimensional and to insure the periodicity conditions on the displacements, the fluctuating part of the cross-sectional strain $\epsilon_{33}^{*}$ has to be null. The condition $\Delta \Sigma_{33}=0$ corresponds to a macroscopic plane stress condition with a non-zero uniform strain component in the cross direction: $\epsilon_{33}=E_{33} \neq 0$. A tolerance of $\eta=10^{-5}$ was taken for the calculations.

The homogenised macroscopic results are represented in the figure 10. One can observe the effect of the plastic yielding of the sedimentary matrix on the global response of the material. The behaviour is clearly non-linear and shows two different trends during the shearing: a first one with the deviator stress value under approximately $0.5 \mathrm{MPa}$ and a second one beyond $0.5 \mathrm{MPa}$. The second part corresponds to the moment when an important part of the local points of the soil matrix yields. The evolution of the fraction of yielding pixels over each entire phase in the figure 11 shows that the soil matrix yields more quickly than the gas hydrates here. Indeed, between $0.62 \mathrm{MPa}$ and $0.83 \mathrm{MPa}$ of the deviator stress values, the fraction of yielding pixels over the soil material increase from $1.49 \%$ to $28.11 \%$ (figures $11 \mathrm{~b}$ and $11 \mathrm{c}$ ).

There are three stages in the behaviour of the unit cells when we look at the figure 11: an elastic part when the deviator $\mathrm{q}$ is below $0.5 \mathrm{MPa}$, the yielding of the soil matrix 


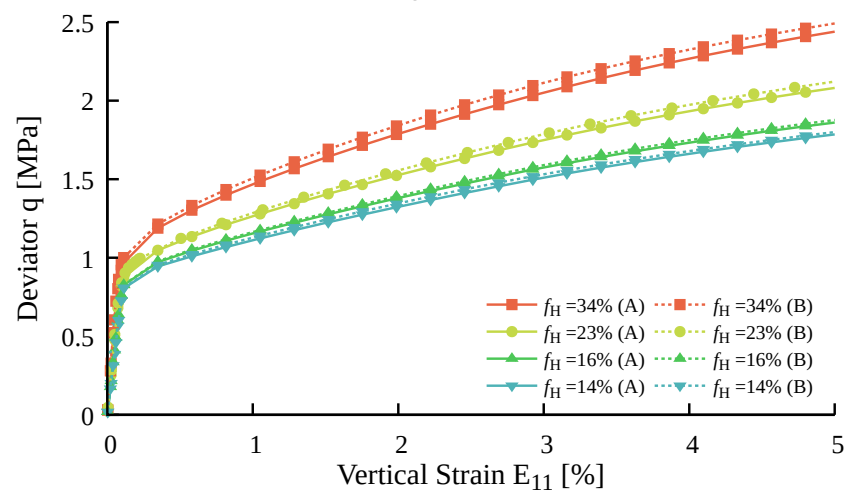

Fig. 10. Macroscopic stress-strain responses of fine soils with hydrate veins inclusions for four different values of gas hydrate volume fractions $\left(f_{\mathrm{H}}=14 \%, f_{\mathrm{H}}=16 \%, f_{\mathrm{H}}=23 \%\right.$ and $\left.f_{\mathrm{H}}=34 \%\right)$, at a resolution of 256 pixels.

pixels when $\mathrm{q}$ is between 0.5 and $1.0 \mathrm{MPa}$ and finally the yielding of the gas hydrate pixels while all pixels of the soil matrix phase exhibit a hardening behaviour when $\mathrm{q}$ is above 1.0 MPa. The distinction between the last two parts of the macroscopic response is not clearly visible in the stress-strain curve of figure 10 though, as the pixels of the gas hydrate phase represent a small fraction of each whole unit cell: $34 \%$ at the maximum. Locally the gas hydrate phase reaches the perfectly plastic regime characterized by a constant value of deviator stress but the hardening behaviour of the soil matrix pixels prevails as they representing $76 \%$ volume fraction of the unit cell at the minimum. Figure 11 also shows that the gas hydrates have an influence on the point when $100 \%$ of the soil matrix pixels have reached the plastic regime. An increase of the gas hydrate volume fraction seems to delay the yielding of the soil matrix as there are more gas hydrates pixels to support the loading.

\section{CONCLUDING REMARKS}

We applied a numerical homogenisation method to gas hydrate bearing sediments to overcome the experimental difficulties usually met in the study of these soils. The chosen FFTbased homogenisation method can help model the effective mechanical behaviour of different types of materials defined by complex microstructures that can be obtained from real images, thus avoiding the multiplication of experiments. This is an advantage especially for the modelling of fine sediments as it is still difficult to form gas hydrates in fine grained soils in laboratory. We used the numerical method on a real image of fine sediments from micro CT scans. We investigated the impact of the choice of threshold value on the results and in our case, with two different threshold values this impact was quite low. More elaborate methods can be used to determine the threshold value. As for granular soils, examples were given for schematic unit cells formed with only few circular grains being surrounded by either cementing or load-bearing gas hydrates.

The examples gave a good insight of the advantages and potential of the method. In particular, the use of the FFT-based methods is less limited when applied to fine-grained sediments in comparison with coarse-grained sediments as the coarsegrained sediments have a more discrete microstructure with larger stiffness ratios between local materials because of the voids. Several applications to gas hydrates are possible such as multi-scale modelling or developing macroscopic laws based on microstructural particularities.

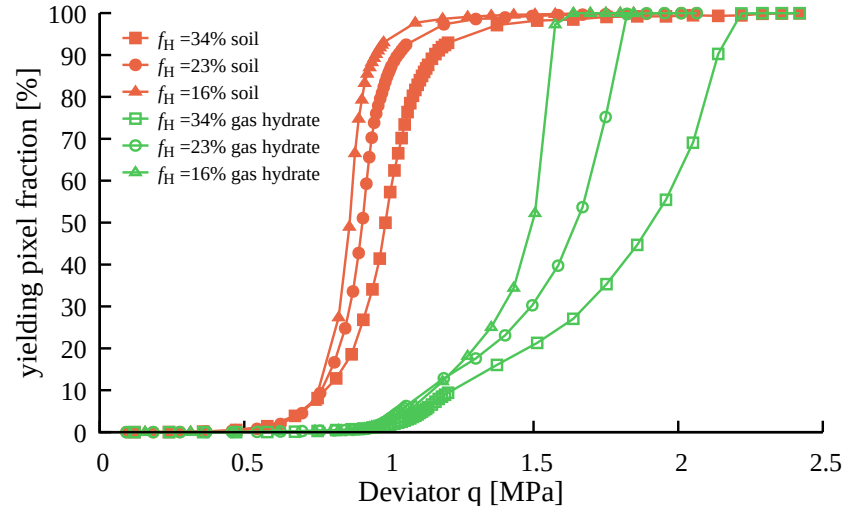

(a)

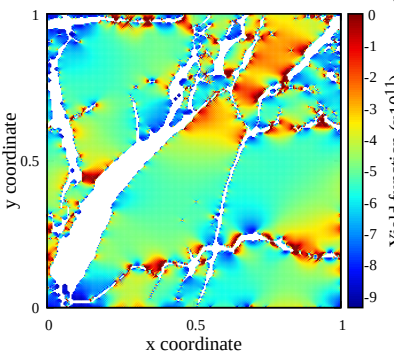

(b) $\mathrm{E}_{11}=0.07 \%, \mathrm{q}=0.62 \mathrm{MPa}$

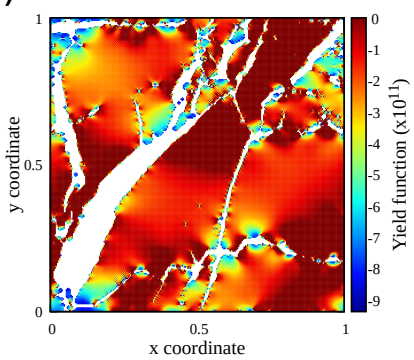

(c) $E_{11}=0.11 \%, q=0.83 \mathrm{MPa}$
Fig. 11. (a) Evolution of the fraction of yielding pixels for each phase; (b) and (c) represent the yield function distribution over the soil phase (Cam Clay) of the microstructure with $16 \%$ of gas hydrate volume fraction at two different stages of the shearing (resolution of 256 pixels; threshold value B). The yield function is the equation of the yield surface: negative values correspond to the elastic regime while zero corresponds to the yield regime.

\section{ACKNOWLEDGEMENTS}

The authors are grateful to S. Brisard for his advice and enriching discussions on FFT-based homogenisation methods. They would also like to express their great appreciation to the French National Research Agency for funding the present study, which is part of the project HYDRE "Mechanical behaviour of gas hydrate bearing sediments" - ANR-15-CE060008 .

\section{REFERENCES}

Al Mahbub, A. \& Haque, A. (2016). X-ray Computed Tomography Imaging of the Microstructure of Sand Particles Subjected to High Pressure One-Dimensional Compression. Materials 9, No. 11, 890, doi:10.3390/ma9110890.

Balay, S., Abhyankar, S., Adams, M. F., Brown, J., Brune, P., Buschelman, K., Dalcin, L., Dener, A., Eijkhout, V., Gropp, W. D., Karpeyev, D., Kaushik, D., Knepley, M. G., May, D. A., McInnes, L. C., Mills, R. T., Munson, T., Rupp, K., Sanan, P., Smith, B. F., Zampini, S., Zhang, H. \& Zhang, H. (2019). PETSc Web page. https://www.mcs.anl.gov/petsc.

Brisard, S. (2018). moisan2011. https://github.com/ sbrisard/moisan2011.

Brisard, S. \& Dormieux, L. (2010). FFT-based methods for the mechanics of composites: A general variational framework. Computational Materials Science 49, No. 3, 663-671, doi:10.1016/ j.commatsci.2010.06.009.

Chaouachi, M. (2016). Microstructure of gas hydrates in sedimentary matrices. Ph.D. thesis, University of Göttingen.

Ebinuma, T., Kamata, Y., Minagawa, H., Ohmura, R., Nagao, J. \& Narita, H. (2005). Mechanical properties of sandy sediment containing methane hydrate. In The Fifth International Conference on Gas Hydrates, Trondheim, Norway.

Gélébart, L. \& Mondon-Cancel, R. (2013). Non-linear extension of FFT-based methods accelerated by conjugate gradients to evaluate 
the mechanical behavior of composite materials. Computational Materials Science 77, 430-439, doi:10.1016/j.commatsci.2013.04 046.

Hyodo, M., Yoneda, J., Yoshimoto, N. \& Nakata, Y. (2013). Mechanical and dissociation properties of methane hydrate-bearing sand in deep seabed. Soils and Foundations 53, No. 2, 299-314, doi:10.1016/j.sandf.2013.02.010.

Hyodo, M. O., Hyde, a. F. L., Nakata, I., Yoshimoto, N., Fukunaga, M., Kubo, K., Nanjo, Y., Matsuo, T. \& Nakamura, K. (2002). Triaxial Compressive Strength of Methane Hydrate. The Twelfth International Offshore and Polar Engineering Conference 3, 422428.

Kato, A., Nakata, Y., Hyodo, M. \& Yoshimoto, N. (2016). Macro and micro behaviour of methane hydrate-bearing sand subjected to plane strain compression. Soils and Foundations 56, No. 5, 835847, doi:10.1016/j.sandf.2016.08.008.

Le, T.-X., Aimedieu, P., Bornert, M., Chabot, B., Rodts, S. \& Tang, A. M. (2019). Effect of temperature cycle on mechanical properties of methane hydrate-bearing sediment. Soils and Foundations 59, No. 4, 814-827, doi:10.1016/j.sandf.2019.02.008.

Masui, A., Haneda, H., Ogata, Y. \& Aoki, K. (2005). The effect of saturation degree of methane hydrate on the shear strength of synthetic methane hydrate sediments. In The 5th International Conference on Gas Hydrates, Trondheim, Norway, pp. 657-663.

Mcknight, R. E. A., Moxon, T., Buckley, A., Taylor, P. A., Darling, T. W. \& Carpenter, M. A. (2008). Grain size dependence of elastic anomalies accompanying the $\alpha-\beta$ phase transition in polycrystalline quartz. Journal of Physics: Condensed Matter 20, No. 075229, doi:10.1088/0953-8984/20/7/075229.

Michel, J. C., Moulinec, H. \& Suquet, P. (2000). A computational method based on augmented lagrangians and fast fourier transforms for composites with high contrast. CMES - Computer Modeling in Engineering and Sciences 1, No. 2, 79-88.

Miyazaki, K., Masui, A., Sakamoto, Y., Aoki, K., Tenma, N. \& Yamaguchi, T. (2011). Triaxial compressive properties of artificial methane-hydrate-bearing sediment. Journal of Geophysical Research: Solid Earth 116, No. 6, 1-11, doi:10.1029/ $2010 J B 008049$.

Moisan, L. (2011). Periodic Plus Smooth Image Decomposition. Journal of Mathematical Imaging and Vision 39, No. 2, 161-179, doi:10.1007/s10851-010-0227-1.

Monchiet, V. \& Bonnet, G. (2013). Numerical homogenization of nonlinear composites with a polarization-based FFT iterative scheme. Computational Materials Science 79, 276-283, doi:10. 1016/j.commatsci.2013.04.035.

Moulinec, H. \& Suquet, P. (1994). A fast numerical method for computing the linear and nonlinear mechanical properties of composites. Comptes Rendus de l'Académie des Sciences Série II 318, 1417-1423.

Moulinec, H. \& Suquet, P. (1998). A numerical method for computing the response of composites with periodic microstructure. Comput. Methods Appl. Mech. Engrg. 157, 69-94, doi:10.1016/ S0045-7825(97)00218-1.

Rees, E. V., Priest, J. A. \& Clayton, C. R. (2011). The structure of methane gas hydrate bearing sediments from the Krishna-Godavari Basin as seen from Micro-CT scanning. Marine and Petroleum Geology 28, No. 7, 1283-1293, doi:10.1016/j.marpetgeo.2011.03. 015 .

Santamarina, J. C., Dai, S., Terzariol, M., Jang, J., Waite, W. F., Winters, W. J., Nagao, J., Yoneda, J., Konno, Y., Fujii, T. \& Suzuki, K. (2015). Hydro-bio-geomechanical properties of hydrate-bearing sediments from Nankai Trough. Marine and Petroleum Geology 66, 434-450, doi:10.1016/j.marpetgeo.2015.02.033.

Sloan, E. D. \& Koh, C. (2007). Clathrate hydrates of natural gases. Third edn., CRC Press.

Soga, K., Ng, M., Lee, S. \& Klar, A. (2006). Characterisation and engineering properties of methane hydrate soils. In Characterisation and Engineering Properties of Natural Soils (Tan, T., Phoon, K., Hight, D. \& Leroueil, S., eds.), London: Taylor and Francis, pp. 2591-2642, doi:10.1201/NOE0415426916.

Torisu, S. S., Pereira, J.-M., De Gennaro, V., Delage, P. \& Puech, A (2012). Strain-rate effects in deep marine clays from the Gulf of Guinea. Géotechnique 62, No. 9, 767-775, doi:10.1680/geot.12. OG.015.
Waite, F., Santamarina, J., Cortes, D., Dugan, B., Espinoza, D., Germaine, J., Jang, J., Jung, J., Kneafsey, T., Shin, H., Soga, K., Winters, W. \& Yun, T. (2009). Physical properties of hydratebearing sediments. Reviews of Geophysics 47, No. 4, 1-38, doi: 10.1029/2008RG000279.

Yoneda, J., Kida, M., Konno, Y., Jin, Y., Morita, S. \& Tenma, N. (2019). In Situ Mechanical Properties of Shallow Gas Hydrate Deposits in the Deep Seabed. Geophysical Research Letters 46, No. 24, 14459-14468, doi:10.1029/2019GL084668.

Yoneda, J., Masui, A., Konno, Y., Jin, Y., Egawa, K., Kida, M., Ito, T., Nagao, J. \& Tenma, N. (2015). Mechanical behavior of hydrate-bearing pressure-core sediments visualized under triaxial compression. Marine and Petroleum Geology 66, 451-459, doi: 10.1016/j.marpetgeo.2015.02.028.

Yu, F., Song, Y., Liu, W., Li, Y. \& Lam, W. (2011). Analyses of stress strain behavior and constitutive model of artificial methane hydrate. Journal of Petroleum Science and Engineering 77, No. 2, 183-188, doi:10.1016/j.petrol.2011.03.004.

Zeman, J., Vondřejc, J., Novák, J. \& Marek, I. (2010). Accelerating a FFT-based solver for numerical homogenization of periodic media by conjugate gradients. Journal of Computational Physics 229, No. 21, 8065-8071, doi:10.1016/j.jcp.2010.07.010. 